\title{
Analysis of Solar Cells Efficacy with Imbedded Layer of Spherical Plasmonic Nanoparticles
}

\author{
S.A. Reshetov ${ }^{\text {a }}$, Yu.V. Vladimirova, and V.N. Zadkov \\ International Laser Center and Faculty of Physics M. V. Lomonosov Moscow State University, Leninskie Gory, \\ Moscow, 119991, Russia
}

\begin{abstract}
The scattered sunlight absorption efficacy by a solar cell with imbedded layer of spherical plasmonic nanoparticles is simulated versus the parameters of the imbedded particles, the material they are made of, their density and location in the polymeric buffer layer inclusive. It was shown that the embedded plasmonic nanoparticles cause an increase of the relative efficacy of an organic solar cell with the P3HT:PCBM photosensitive layer and the PEDOT buffer layer of up to $10-20 \%$. This increase depends also on the angle at which the sunlight shines the solar cell, which was also studied in detail.
\end{abstract}

Key words: nanoplasmonics, solar cell, photovoltaics.

The development of the alternative sources of energy such as solar photovoltaic devices and others is one of the urgent problems of our society. In the field of photovoltaics the primary tasks are to increase the solar cells efficacy and significant reduction in their price. For the crystalline silicon solar cells, the problem of increasing their efficacy was largely solved, however they are still very expansive. By contrast, organic solar cells are rather cheap in production, but have quite low efficacy so far [1]. That is why the development of various methods that allows to increase the organic solar cells efficacy is of prime importance [2].

One of such methods of raising the organic solar cells efficacy is the use of plasmonic nanoparticles, which are embedded into the solar cell. Each nanoparticle serves then as a nanoantenna allowing concentrating the incident electromagnetic field in the necessary area and thereby increases the efficiency of the solar cell [3]. Such nanostructures are already used in the silicon solar cells, but technologies of the plasmonic nanostructure deposition are very expensive and laborious.

In this paper we provide a detailed analysis of the efficacy of the solar energy absorption by the solar cell with embedded plasmonic nanoparticles depending on the parameters of the nanoparticles and their coverage. This analysis was made in the quasistatic approximation and for the case of scattered incident sunlight. We assume that the spherical metal nanoparticles are embedded in the buffer layer that is deposited on the photosensitive layer and we the coverage density of the nanoparticles is selected in a way to avoid interaction in between the particles.

The redistribution of the incident solar field energy in the solar cell was calculated with the help of the epsilon method of solving the Maxwell's equations [4]. The respective analysis of the efficacy of embedding the plasmonic nanoparticles with various parameters of the nanoparticles and their

${ }^{\mathrm{a}}$ Corresponding author: sa.reshetov@physics.msu.ru 
coverage in the solar cell is based on the computer simulation of the respective solar cells on the supercomputer "Lomonosov" [5] using the MPI programming technology.

Our computer simulation results show that the increase of the solar cell efficacy with embedded plasmonic nanoparticles was observed at all sets of parameters we used in our calculations, but the maximal efficacy is observed for the silver and gold nanoparticles, which plasmonic resonances lie in the optical part of the spectrum. We also demonstrate an advantage of using a layer of silver nanoparticles embedded in the solar cell, which work also in the short-wave spectral range. Calculations also show that the relative efficacy depends on the angle of the incident light and the cell's plane. Obviously, the maximal efficacy is observed at the normal falling of light at the cell's surface, however at the large angles we also observe the significant increase in efficacy. This is essential for operating the organic thin-film solar cells in a scattered light. The calculated spectral characteristics of the layer with embedded nanoparticles allow estimating the efficacy of the coverage for arbitrary photosensitive layers reducing thereby efforts towards search for the optimal solar sell characteristics.

\section{References}

1. Min Gu, Zi Ouyang, Baohua Jia et al., Nanophotonics $1235-248$ (2012)

2. Z. Tang, W. Tress and O. Inganäs, Materials today, 17, 8, 389-396 (2014)

3. L. Novotny, B. Hecht, Principles of Nano-Optics, (Cambridge University Press, 2012)

4. M.S Agranovich, B.Z Katsenelenbam, A.N. Sizov, N.N Voitovich, Generalized Method of Eigenoscillations in Diffraction theory,(Wiley, Berlin, 1999)

5. V.V. Voevodin, S.A. Zhumatiy, S.I. Sobolev et al., Open Systems J., 7, 36-39 (2012) 